\title{
Perception of Health Workers about Barriers Impacting Organ Donation in Qatar: A Focus Group Study
}

\author{
Tulika Mehta Agarwal ${ }^{1}$, Rajvir Singh ${ }^{1 *}$, Ayman El Menyar ${ }^{1}$, \\ Hassan Al Thani ${ }^{1}$, Yousuf Al Maslamani ${ }^{1}$, Ahammed Abdulla \\ Mekkodathil $^{1}$ \\ ${ }^{1}$ Hamad Medical Corporation, Doha, Qatar \\ *Corresponding author: Rajvir Singh: rsingh@hamad.qa
}

\section{Abstract:}

Citation: Agarwal T.M., Singh R., Menyar A.E., Thani H.A., Maslamani Y.A., Mekkodathil A.A. (2018) Perception of Health Workers about Barriers Impacting Organ Donation in Qatar: A Focus Group Study. Open Science Journal 3(1)

Received: $22^{\text {nd }}$ October 2017

Accepted: 14 $4^{\text {th }}$ December 2017

Published: $2^{\text {nd }}$ January 2018

Copyright:@ 2018 This is an open access article under the terms of the Creative Commons

Attribution License, which permits unrestricted use, distribution, and reproduction in any medium, provided the original author and source are credited.

Funding: NPRP 7-965-3-247, Qatar National Research Fund (QNRF), Qatar

Competing Interests: The author have declared that no competing interests exists.

Objectives: To understand perception of healthcare workers about factors acting as barriers to organ donation and transplantation process in Qatar.

Subjects and Methods: A phenomenological approach with the help of focus group discussions was used to fulfill the objectives of the research. Participants were healthcare workers working as volunteers in organ donation campaigns or playing a crucial role in the process of organ donation or transplantation in Qatar. They were selected purposively to participate in the discussions. Openended questions were asked during the audio-recorded discussions. Transcripts were coded and assessed using NVivo software. Results were analyzed based on the Theory of Planned Behavior to explore intentions shaping an individual's donation decision.

Results: Lack of awareness and information about the process of organ donation were found to be the root cause barriers for both deceased and living organ donation in Qatar whereas; family, religious and community influences, and an incongruous attitude towards organ donation are the main factors influencing organ donation registration and family consent for deceased donation. Conclusion: Improving awareness and accurate information about organ donation could play significant in increasing deceased and living organ donation in Qatar. However, factors impacting family consent, which was found to be the most prominent reason for gap between availability and utilization of organs, need to be further researched. 
Keywords: Organ Donation, Focus Group Discussion, Healthcare Workers, Barriers, Qatar

\section{Introduction}

According to World Health Organization (WHO), shortage of organs for transplantation is essentially a 'universal problem' with variable impacts based on socio-cultural diversity [1].

Challenges for organ donation and transplant in Qatar are unique due to diverse socioeconomic and multiethnic population. These challenges relate to either registration or actual donation process, and are reflected in the form of barriers to organ donation.

The most common type of donation prevalent in the middle-eastern countries is living donation [2]. Similar trend for living donation is prevalent in Qatar [3]. However, there is significant potential for cadaveric donation which is yet to be harnessed for reducing the gap between demand and supply of organs. One of the important reasons why cadaveric donation is still not completely accepted in the middle-east is that the public as well as the medical community lacks awareness about the importance of organ donation and transplant [2].

Underutilization of available organs in Qatar has been observed due to complex problems arising at various stages of the donation process, particularly obtaining consent for donation as is evidenced in several studies. In 2012, of 116 recorded brain death cases in Qatar, organs were retrieved and utilized for transplantation only in 5 cases. Low consent rate was reported as one of the major factors affecting organ transplantation [4].

Barriers to organ donation have been well-researched world over and reasons for low rate of organ donation have been outlined in these studies. Researches in America, Europe and Australia have outlined that lack of knowledge, bodily concerns, ethnic, religious, community and family beliefs and values, uncertainties regarding religious permissibility, conflict between one's own desire and family values, desire for reciprocal benefits are some of the reasons that impact an individual's organ donation decision and act as barriers to the process [5-6]. However, the body of knowledge in context of Organ donation and Transplantation in Qatar is minimal. The only study that researched barriers affecting organ donation in Qatar was published in the year 2006 [7]. The organ donation program started gaining prominence in Qatar only after the Doha Donation Accord was signed in 2009. Hence, a need for a more updated study on this subject was identified and funded as a National Priority Research Project through Qatar National Research Fund.

Expatriate residents in Qatar constitute the largest part of the organ donation pool. As per the Director of Center for Organ Transplant at Hamad Medical Corporation, Qatar, nearly 99 percent of the organ donors in Qatar are expatriates [8]. A need was identified for understanding why people from some communities donate less than others.

There are rising numbers of cases with other chronic diseases such as type 2 diabetes (nearly $16 \%$ of the adult Qatari population is diabetic) and hypertension that largely contribute to the increasing demand of kidney transplant in Qatar 
[9]. Therefore, it becomes very important to understand the barriers that affect organ donation in Qatar that will help to address the demand-supply gap and plan strategies to increase availability of organs for transplantation.

Health care workers working in the field of organ donation and transplant are in the front line when it comes to experiencing the challenges associated with these processes. However, most of the studies internationally that have taken these health care workers as subjects have focused on their knowledge about organ donation and their willingness to donate. There is no study in the region which focusses on perspective of Healthcare workers towards the process of organ donation and the barriers to organ donation based on their interaction with donors or recipients. This study is unique as it uses a phenomenological approach to understand the barriers to organ donation using focused group discussions with healthcare workers.

\section{Subjects and Methods}

Five focus group discussions were conducted with 33 participants who were working in organ donation or transplantation process in Qatar. They were grouped homogenously based on their roles as surgeons, coordinators \& campaign educators. The participants varied in ethnicities and gender. Participants were selected purposively and recruited using emails and phone calls. During the phone calls they were informed that the interviews would be conducted by a female moderator who will be recruited externally for the study and would that the discussions would be audio recorded for accuracy of data. They were able to converse in English and provide consent. Around 4 people dropped out from the study due to engagement as a donor who needed to be transplanted became available on the same day that was scheduled for the focus group discussion. However, repeat interviews could not be carried out with these individuals due to their unavailability.

\section{Data collection and Analysis}

The interview guides were semi structures constituting of open-ended questions were prepared by the research team after extensive literature reviews and inputs from experts in the field. The questions were role-based and standardized for each group.

The discussions were moderated in English by an experienced and unbiased moderator who was working in the capacity of 'Project Manager' for conducting this study and is the first author of this study. The moderator was a Clinical psychologist by profession, female, aged 36 and held a Ph.D degree in Psychological interventions besides being experienced in conducting group discussions and group training for over 10 years. The interviewer had no prior relationship with any of the participant of the focus group discussion.

Participants provided as well as read out verbal informed consent to participate in this study which included introduction of the study, how the data would be kept secure and utilized. Participation after listening to the consent 
indicated agreement to participate as was informed to the participants in the consent.5. Along with the moderator, research assistants and transcribers were also present for taking notes of the discussions which were audio recorded. As discussed within the research team, the discussion was closed when saturation point was reached for each question. Feedback about the session was taken from participants after completion of each session. Every session was followed by a debriefing session for quality improvement.

The focus group discussions were organized between March -April 2015 in a neutral venue at the Medical Research Center and lasted for 60 minutes and were audio recorded. Audio-recorded data was transcribed by transcribers and checked for accuracy by research team members. However, they were not sent back to the participants for comments. The unstructured data was classified, sorted and arranged to identify the trend for evidences of organ donation barriers using the NVivo statistical software package. The coding of the data was primarily done by two researchers, each of who provided description for the codes made. The coding of the two researchers were compared and consolidated. These codes were checked for accuracy by the other team members.

The findings of the present study have been analyzed and discussed based on Icek Ajzen's 'Theory of Planned Behavior' (TPB) [10]. This theory is considered as one of the most influential and popular conceptual frameworks to study the human actions. According to TPB, human behavior is guided by three considerations: (a) beliefs about the likely consequences (behavioral beliefs), (b) beliefs about the normative expectations of other people (normative beliefs), and (c) beliefs about the presence of factors that may further or hinder performance of the behavior (control beliefs). These three beliefs lead to intentions about the behavior, which combined with actual controls lead one to perform or avoid the behavior in consideration.

\section{Results}

The barriers and recommendations that were brought out during the focus group discussions are presented in the following sections.

\section{Barriers}

The main themes and sub-themes that emerged from the transcribed data were:

1. Personal Barriers: incongruous attitude towards organ donation, lack of knowledge, lack of clarity about religious stance, mistrust in system, fear of death;

2. Social Barriers: family, religion, peer influence, culture \& ethnicity;

3. Others: lack of a supporting law for voluntary donation, challenges faced during organ donation campaigns and in getting family consent for deceased donation. 


\section{1) Personal Barriers:}

(i) Attitudinal barriers of individuals attending organ donation campaigns:

a) Apathy: People tended to avoid the campaigns with various excuses, or agreeing to register just to get the donor card without trying to understand details. Refer Table 1, item 1 for illustrative quotes.

Table 1: Illustrative Quotations from Focus Group Discussions

\section{\# Sub Theme Illustrative Quotations}

1 Apathy for the subject of Organ donation SP2/TE4: And whatever you are saying, anything for explaining to them. They are ignoring everything. They don't want to hear

SP7/TE8: They are running away. Few are run away from our talk itself.

2 Lack of interest in SP7/TE3: Practicality. So, they are thinking that everything has a price. Sad to say voluntary donations that but, you know, only few people are very, you know, welcoming the idea of voluntary $100 \%$.

3 Lack of trust on SP2/TE4: They are asking _one question.... you are mutawa? how can you are what's being communicated saying... what is the main purpose? how... from where did you get this Fatwa? They are asking like that.

4 Mistrust in System

SP8/UC1: They don't understand what organ donation is, so the only thing they think is that, you know, some other countries where they procure organ without their consent. They think like that they are trying to take the organs from them. The first thing they say is that no we want the body to come back. That's the main issue with them. They say no we don't want the organs to be...[Donated]

5 Impact of country of origin
SP1/TE1: For some people, this is like the first thing they have heard, I mean, maybe it is not a common thing in their country. Because for me, I met some people from my country. It is not a common thing for organ donation in my country, so it is like a new thing, even though, like... we already explained, we tried our best to give information but still... yeah... they still need, I think, to consider about it and need to decide before they register.

6 Lack of information about living donation after effects

SP3/TOR3: But the thing that how the work... how will be affecting my work after donation.

SP3/TOR1: And if she's a woman, I can get pregnant? I have baby. I can live with 1 kidney? Are you sure?

7 Lack of Information about transplant procedures in Qatar
SP1/TE5: Even the nationals the Qatari people, they do not... much of the people do not know this thing is going on, there is campaign going on or there is a facility. 


\section{\# Sub Theme}

8 Religion related ambiguity

\section{Illustrative Quotations}

SP1/TE5: They are willing but they do not know is it right or wrong in terms of religious, so they are afraid. They are telling who... if there's any, you know... for sure things that is written that he can do this, this, this. So, it is okay or it will come back to me. I'll be doing the wrong thing that is it.

9 Family members dissuading registration
SP1/TE3: While they are coming next day, they are contacted by families. The family members are saying, "Oh no, this is not good to do. No, you cannot do this. Don't do that." Like that they are saying. And next day, they are coming, "We don't want, our families not allowed to give this. So, please cancel this card. Cancel." So many, so many peoples are like that.

10 External influence in Family consent

SP6/HR5: Absolutely, sometimes what happens is that you relate a news and they are there shocked and so it takes more than 1 conversation to get the consent because sometimes they get into a meeting with the family members, sometimes they talk to the religious person...

11 Peer impacting acceptance of brain death

SP3/TOR2: Especially, he is calling his friends or his relatives there and says where he is? Still, he is in the bed and he is still breathing... And still in... still taking care in the hospital. Then he is saying how this can be? So, he is breathing and you told me that he's dead.

12 Language impacting family consent

SP8/UC3: From here, you call the... whoever is calling from here, he's calling in English or maybe in one language and those people are poor. They don't understand. There is a lot of miscommunication between the person telling them and what they're listening.

13 Fear of Death SP1/TE6: Sometimes, they will like care if we mention the after death, if they listen or hear this word, they told us ah it is okay, no need, I have to go, especially Arab,,, Arab people. Like, because _i am from Egypt, like Egyptian and like Jordanian, and I think some societies like that they are afraid, afraid to do that.

14 Impact of community SP5/OR5: But that's why in donor registry, the smallest registration is from Arabic countries.

SP2/TE4: Qatari people, they are also not registering.

SP7/TE7: They are in Nepal or Bangladesh, they are

telling our culture is not allowing to do this one.

15 Remote locations of family members
SP8/UC1: Sometimes, the families cannot be reached because especially Nepal, they stay in the higher mountain area and they don't have the mobiles to reach them. It's very difficult. So, we try to make a call in the Katmandu area then there somebody try to pass the message, so it takes a lot of time before we come to a conclusion that 


\section{\# Sub Theme Illustrative Quotations}

this organ is to be taken or not. That's a major issue for us.

16 Lack of Supporting SP5/OR3: There is no supporting law to take that organs from the [individual]. Law

SP\#/ TE\# / HR\# / TOR\# / UC\# / OR\#: Focus group participants' coding

b) Lack of trust: Some participants were unable to trust the educators regarding the acceptance of organ donation by their own respective religions even when supporting documents were provided. Refer Table 1 , item 3 .

c) Incentives: Individuals approaching organ donation campaigns showed reluctance towards voluntary donations. They believed that organ donors should be given incentives for registering as donors. Examples of incentives that people expected include priority in emergency as well as regular medical services, free-of-cost transplant if any of their family members in their home countries required the same, and freeof-charge health check-ups and treatment. Refer Table 1, item 2.

(ii) Lack of knowledge, clarity and education: Some people did not have knowledge about the registration process such as locations for registration, time taken in the registration process, or post-registration process such as access in case of death in home country, applicability of consent from family members. Some people said that their decision would be based on the knowledge of who would be the recipient. Living donors lacked clarity on how organ donation would affect their work efficiency, job status, ability to conceive and take care of children, life span, etc.

Lack of media coverage also contributed to people's lack of knowledge about organ donation program in Qatar. Participants expressed that lack of information and clarity raised doubts, made people fearful and apprehensive, as well as made it more difficult for family members to understand the concept of organ donation and give consent. Refer Table 1, items 6 and 7 .

(iii) Religious ambiguity: Participants reported that some people exhibited ambiguity around religious righteousness concerning organ donation making them apprehensive. For instance, while being charitable is considered to be very important in Islam, it is also considered important to bury an intact dead body. Some leaders of the same religion support organ donation claiming that it aligns with the religion while others reject it completely believing it is against the religion.Since some people chose to selectively follow certain religious beliefs more firmly.Hence, religion as a factor acted as both a barrier as well as a promoter for organ donation.Some people refused to donate certain parts of their body such as eyes while others completely refused registration or consent for organ donation to avoid mutilation of body per their religious beliefs. Refer Table 1, item 8 . 
(iv) Mistrust in system: Many participants indicated that lack of trust toward system was a significant factor both while registering and while seeking family consent for organ donation. Individuals' belief of not being taken care of in a medical emergency if they registered as donors made them refrain from registering. Next of kin when approached for consent often felt that their family member's organs were being harvested deceitfully. As per the participants, such mistrust was greater in people from countries where organs were often harvested deceitfully or without consent, such as India, Philippines and Nepal. Refer Table 1, items 4 and 5.

\section{2) Social Barriers}

i. Family and peers: The discussion brought out how family and friends of individuals acted as barriers to organ donation, during registration as well as for consent for deceased donation.

Several participants reported that female spouses often dissuaded willing husbands from registering for organ donation. In several cases, disapproval from family members after one had registered as a donor resulted in cancelation of registration. Some refused to register because they were sensitive about their family's predicament if approached for consent after death. Family often denied consent when the decision maker in a family and legal heir to consent for donation were separate individuals. Refer Table 1, item 9.

People were also influenced by peers' views about organ donation. Sometimes single men living together in camps came to cancel their registration in groups. Refer Table 1, item 11.

ii. Culture and ethnicity: As per the participants, culture and ethnicity were considered amongst the most important barriers to organ donation affecting both registration decision and family consent. People's decisions were often influenced by views of a religious leader and community. Some communities have a belief of intact burial hence there is refusal to donate parts of their body as it could disfigure and show disregard for the deceased individual. Participants indicated that people from Arabic nations such as Egypt and Jordan, and Philippines showed greater reluctance to register as donors because they lacked familiarity and acceptance for organ donation in their culture, and had taboo associated with discussing death. Filipino community also showed a fear of bodily disfigurement and procedures. Individuals from Bangladesh and Nepal often indicated that their culture did not support organ donation.

Very few Qatari citizens showed interest in volunteering for organ donation registration or stopping at campaign booths. People from western communities seemed to accept the concept of organ donation but did not always stop to register at the organ donation campaigns. However, when approached to register they did not show resistance. Refer Table 1, items 13 and 14. 


\section{3) Other Barriers}

i. Lack of a supporting law for voluntary donation: Participants expressed that the law of Qatar was not supportive of organ donation. They disapproved the fact that there was no law that supports organ extraction from a deceased individual without family consent even when he himself had registered for the donation. Refer Table 1, item 16.

ii. Campaign challenges: Some challenges related to organ donation campaigns indirectly influenced organ donation registrations in Qatar. These were related to less number of staff, language of campaign staff, lack of training to answer queries satisfactorily and campaign infrastructure such as availability of just one card printer in each camp and less space.

iii. Challenges in getting family consent: The highest number of brain death cases who could be potential donors constituted of male workers whose families lived in their native countries. Contacting families living in remote locations, no face-to-face communication, communication gap due to language barriers and lack of knowledge about brain death were important factors affecting family consent for deceased donation. Refer Table 1, item 12.

\section{Recommendations}

i. Campaign incentives: Most educators agreed that incentives in form of small gifts should be provided to all attending campaigns regardless of whether they registered or not. This would help increase footfalls in camps spreading awareness as well as influence registrations.

ii. Incentives for deceased consent: Participants were divided about incentives for deceased consent and more than half opined that providing financial incentives for deceased consent would be unethical. However, at least two participants strongly supported providing incentives (financial or others) to support consenting family of the deceased to help reduce the gap between the demand and availability of organs since this would be a win-win situation for all.

Nevertheless, all participants unanimously agreed that the approach taken by Doha Donation Accord was appropriate wherein after assessment of financial status of the family of deceased, incentives are offered through charitable organizations in Qatar, to support children's education and other needs without any direct financial implication, regardless of consent obtained or not.

i. Law: Participants proposed that if the law was made to be more supportive towards an individual's decision to donate, then family would have to abide by it and would not have the right to override participant's decision. 
ii. Awareness and Information: Participants suggested that along with other efforts to popularize organ donation, doubts related to aftereffects of living donation should also be addressed through media so that people feel more in control when they come up for living donation.

\section{Discussion}

Within the context of the Theory of Planned Behaviour, the results of the present study brought out that "non-donation behavior" of residents of Qatar and their family is influenced by their intentions towards organ donation, feeders as well as actual controls. In case of deceased donation these intentions were highly influenced by an individual's attitude towards organ donation and the normative influences of family, peers, community, religious group or leader. However, in case of living donation there was a greater influence of feeders and actual controls whenever the individual could not donate. Please refer Figs 1 and 2.

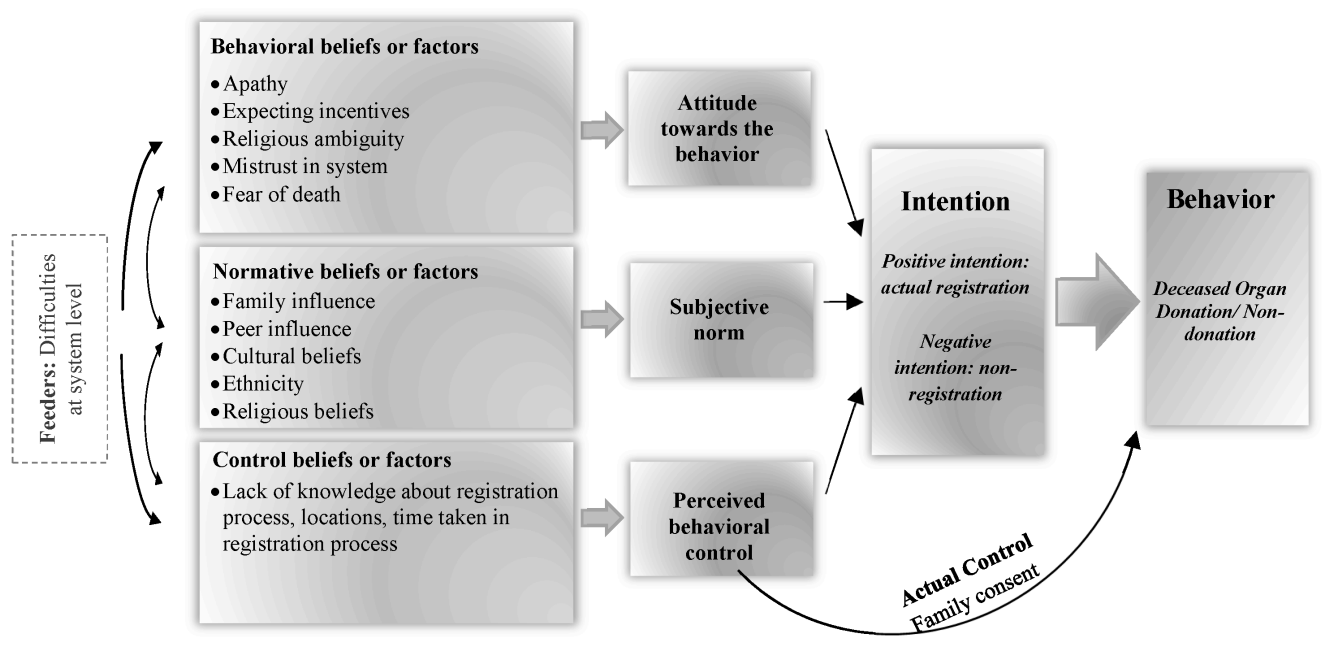

Fig 1. Factors Influencing Deceased Organ Donation based on the Theory of Planned Behavior 


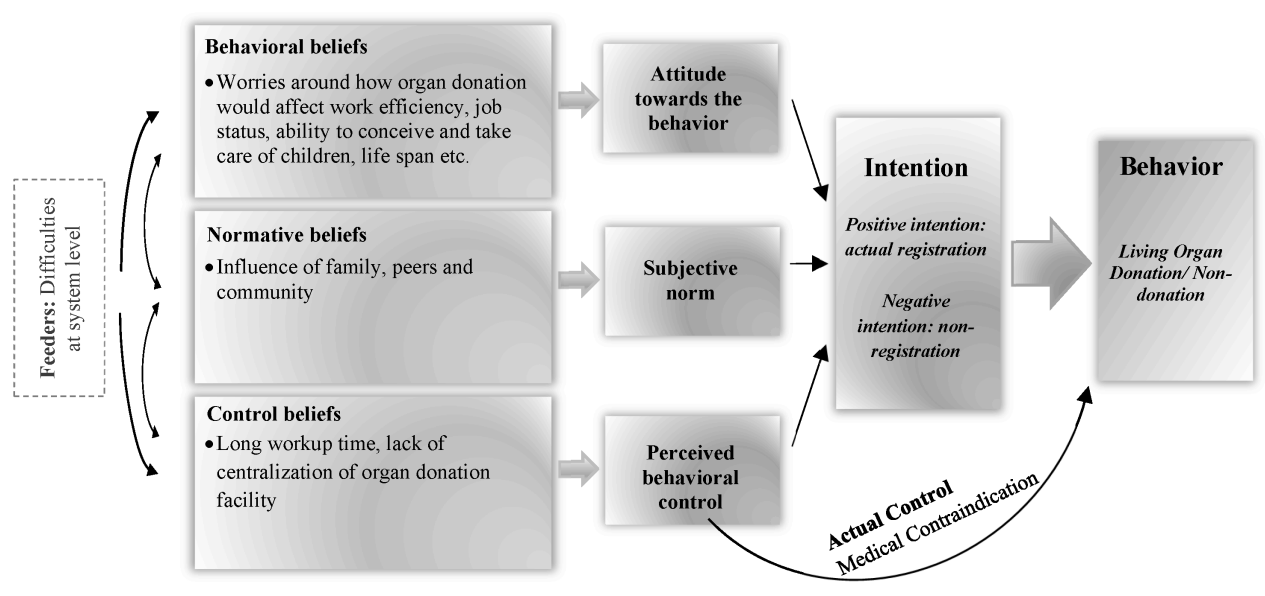

Fig 2. Factors Influencing Living Organ Donation based on the Theory of Planned Behavior

Deceased Donation in the context of Qatar constitutes of registration for organ donation and family consent for the donation. Registration for the deceased donation can happen either in organ donation campaigns or at the organ donation centre. The barriers that were brought out showed that while registering for organ donation, most individual who had families in Qatar gave high amount of importance to how their family would look at their registration decision. At the same time single men living in groups were often seen to be influenced more by the views of peer living with them. These influences were seen both before and after registration. It was sometimes seen that people came back and cancelled their registration after discussion with family or peers.

Community beliefs or ethnic influences were mostly driven by cognitive and affective components of fear or emotionality. People from some ethnicities who rarely came up for registering as deceased donors, often associated death with organ donation and felt fearful about it or believed that bodies of deceased individuals should be buried intact. Several studies show that people from some communities often associate fear of premature death and dying with organ donation $[11,12]$. Another study that came up with similar results, deduced that such bodily concerns could be a result of a cognitive component resulting from fear of organ donation or an affective component relating to damage to body after death [13].

Qatar has a mix of cultures as citizens of several different countries following different religions reside here besides the local Qatari population. However, the main religion practiced in Qatar is Muslim [14]. People in Qatar tended to resort to religious doctrines while deciding about organ donation. However, religious ambiguity often made it difficult for the individuals to trust any information about organ donation that was being provided by educators. It was noticed that in case of ambiguity, individuals consider only those views which were followed in 
their own community as a benchmark for their own views about organ donation. According to a research done in Australia people tend to resort to religion for guidance when they are mostly unfamiliar with organ donation [6]. Intactness of body during burial was found to be an influential belief associated with Muslim as well as Christian religion which impacted their decision negatively.

In keeping with the Theory of Planned Behavior the second biggest factor impacting deceased donor registration is an incongruous attitude towards organ donation. According to TPB, behavioral beliefs shape ones attitude towards the behavior in question and impact intentions \& are related to a certain belief about the outcome of performing the behavior [15]. At several points during the discussion it was noticed that there was an overlap between normative beliefs and behavioural beliefs such that certain normative influences resulting from ones community, ethnicity or culture led one to develop behavioural beliefs that led to incongruous donation attitude. For e.g. People coming from countries where organs were procured without formal consent or by deceit, tended to mistrust organ donation process even in Qatar which was usually transference of learnt fears reflecting in their attitude towards organ donation. Similarly, fear of death in some communities was actually a reflection of an attitude of conflict resulting from negative behavioural beliefs associated with organ donation.

Both the incumbents in a campaign as well as the educators believed that giving incentives during the campaign could increase the registrations for donation. However, it was argued that giving incentives especially in the form of monetary remunerations to consenting families would be unethical as it would be a form of coercion. Often people refused to register when no incentives were assured.

Another cultural factor that was identified was that women were more hesitant in registering for deceased donation and more apprehensive towards organ donation as compared to men in Qatar. Consistent with our findings, the resistive behavior of women was also reported by an NHSBT organ donation study conducted in England [16]. This study showed that in overall $61 \%$ individuals had some reservations towards organ donation and particularly women have significantly more concerns as compared to men. In contrast, an earlier survey-based study from Qatar which measures the attitudes of respondents towards organ donation indicated that generally male respondents $(31.7 \%)$ showed less acceptance for organ donation than females (39.5\%) [7]. This discrepancy could be attributed to the fact that this study measured the attitude towards organ donation without measuring other factors such as normative beliefs and controls which contribute to the intention and finally lead an individual to register or not register as a donor while at the organ donation camp. The third factor according to the Theory of planned behaviour that influences intentions towards the behaviour is Perceived Behavioural Controls. Organ donation campaigns running nearly throughout the year in prime public locations in Qatar have contributed to reducing these perceived challenges associated with the registration process. For e.g. ease associated with access to organ donation information and registration has improved. However, these challenges still continue to exist for those who intend to register for donation at the center for organ donation in Qatar as people still have doubts about where the centre is located and how much time would be spent in doing the registration. Similarly, some infrastructural and other challenges which still 
continue to exist in campaigns such as lack of space, availability of just one printers in each camp for printing registration cards, less number of educators or language barriers, continue created difficulty in the access to organ donation information and increased the waiting time for obtaining registration cards, which often acted as perceived control factors for non-registering individuals.

One of the main factor that acted as an actual control for the individuals' registered for organ donation was family consent. Which means that even after having registered their intent for donation, often actual donation could not be done because of several actual controls such as refusal of family consent or damage to organs due to delay in harvesting. According to the organ donation law in Qatar (Article 13 of Qatari law No 15 Regulating the Human Organs Transfer and Transplantation), actual donation is completely out of the volitional control of the registering individual himself. In case of deceased donation, organs can only be transferred after family consent has been obtained [17]. Therefore, barriers in getting family consent became very important barriers to organ donation in Qatar. As per statistics shown earlier, a large number of both registered as well as non-registered potential donors do not become actual donors as a result of refusal for consent by next of kin.

High influence of significant family members, community and religious beliefs was reported to be impacting the consenting intention. In case of individuals came from a country where organ donation had low acceptance, there was greater tendency for them to refuse the donation. However, even in such a context the increase in number of organ donor registrations within the country could still be considered as an indicator of continuously increasing awareness and acceptance for organ donation.

As per the participants, normative influences have the highest influence on the decision of the bereaved family member who is being approach for consent. However, here the normative influences could be more a result of feeders such as personal lack of knowledge about what is organ donation amalgamated with practices related to organ donation in their home country and lack of information about the prevailing process of organ donation in Qatar. Studies conducted from the viewpoint of various theoretical stances such as TPB and Social Norms Approach (SNA) have found that subjective norms strongly influences the organ donation related behaviors [18, 19]. Another research also supported that the reason behind this could be lack of awareness and knowledge among most individuals regarding organ donation and its process in Qatar [2]. Therefore, it could be assumed that individuals often resort to beliefs or opinions of significant others when it comes to forming intentions in relation to organ donation as they themselves lack awareness or information of the same.

It was also brought out that when individuals came from a country where organ donation had low acceptance, there was greater tendency for them to refuse the donation.

Another important factor that impacts next of kin's refusal of consent was that educators never asked the registering individuals to communicate their donation decision to their family members. This Non- communication acts as a feeder that creates ambiguity in the minds of the family member about what the deceased individual must have wanted and influences organ donation behaviour negatively. An article written in context of the Islamic world also stated that in 
obtaining family consent the biggest challenge is that the members are unaware whether the deceased had an intention to donate organs or not [20].

The attitude of non-consenting family members is also influenced by two additional factors namely lack of awareness of what is brain death and organ donation and lack of knowledge about the scrupulous process of organ donation in Qatar. These combined with feedback of peers who believe the brain dead patient on ventilator is still alive, lead to creating high level of mistrust in the family members towards the system and in turn impact their intentions towards consent negatively.

In case of living donation, negative behavioral beliefs towards organ donation arose out of lack of knowledge about the aftereffects of the donation. When it came to living donation, behavioral beliefs associated with the process and after effects of the donation impacted the attitude of the individual towards the donation and played a dominant role when it came to refusal of consent. These beliefs were fed by their ignorance and lack of knowledge about how donation would impact their health, capacity to work or bear child and life expectancy. According to Ajzen \& Fishbein, external variables will influence the behavior indirectly through the model (TPB) components, by acting as feeders [15]. It was suggested during the discussion.

In case of living donation lack of centralization of organ donation facility acted as a perceived behavioral control for the registered individual and often results in drop-out of living donors. For living donation negative normative influences were not reported during the discussion. This could be an indicator that living donation is more acceptable in this country.

Finally, an actual control impacting living donation is Medical contraindication. Even when a living, consented donor was available, sometimes their organs could not be used for the transplant because of various medical factors which rendered the donor unsuitable for the donation. These factors acted as actual controls and impacted the donation behavior. A considerable number of living donor drop-out, observed at the initial stages, could be related in part related to medical contraindications $[21,22]$.

\section{Conclusion}

Normative influences of family, culture and religion and an incongruous attitude towards organ donation are the primary barriers to organ donation in Qatar which are primarily fed by factors such as lack of awareness and information about the prevailing process of organ donation and transplant, and associated laws. The current biggest challenge in Qatar is family consent. Factors impacting this need to be further explored. Encouraging registering individuals to communicate their donation decision to family could make a positive difference in getting more family consents.

Increasing awareness about organ donation and the process it involves in Qatar could be the most important step to dealing with the various barriers, building a positive attitude towards organ donation and clear the numerous doubts that people have about it. Although, this was a qualitative study using 
focus discussions, it was able to bring out several chalenges to organ donation similar to quantitative studies conducted all over the world $[5,6,7,8,11,13,16]$.

The present study supports the use of an extended TPB in understanding donation-related intentions and behaviors and it could be used in future for predicting organ donation behaviors. TBP helps categorize the main area of impact hence potential targets of interventions to promote organ donation can be devised using this theory. It is believed that in the context of organ donation feeders such as system level issues play an important role in impacting donation and acting as barriers impacting intentions. However, discussion of these was beyond the scope of thispaper.

\section{Acknowledgement}

This study was granted by Qatar Nation Research Fund (QF) under the program NPRP7-965-3-247. Ethical approval was taken from IRB, Medical Research Center, HMC, Doha, Qatar. We appreciate contribution of Medical Research Center, HMC for facilitating the study. We appreciate contribution of Prof. Riadh Fadhil, Director, Qatar Organ Donation Center, HMC for his constant support to the research project team. We also acknowledge the training support provided by Dr. Asmaa Al Khatib, Manager, Oral Health Division Service Development and Health Promotion Primary Health Care Corporation.

\section{Limitations}

Barriers impacting the living donor drop-out and family consent could not be explored completely due to in-adequate information during the discussions. Discussion of system level challenges was beyond the scope of this paper. Actual extent of impact of religion on organ donation behavior couldn't be brought out in the discussions.

\section{Future Direction}

Minimization of living donor's dropouts, counter skewness of gender and ethnic group for participation for organ donations need to be further researched. Factors impacting family consent and ways of improving consent from family members need to be further researched and identified.

The results of this study are based on experiential views of health care workers working in the field of organ donation and transplantation and therefore give an overall picture of organ donation in Qatar. However, they cannot be generalized to the population and there is a need to do a quantitative study on the subject with resident population of Qatar so as to establish the barriers that came up during the focused group discussions. 
Several system level challenges impacting organ donation and transplantation were brought out during the course of the discussions. It is beyond the scope of this paper to discuss these.

\section{References:}

1. Shimazono Y: The state of the international organ trade: a provisional picture based on integration of available information. Bulletin of the World Health Organization 2007; 85(12): 955962.

2. Shaheen FA: Organ donation in the Middle East countries. Ethn Dis 2009;19(1):16-17.

3. Almaslamani Y, Almalki H, Fadhil R, et al: Transplantation in Qatar. Transplantation DOI: 10.1097/TP.0000000000001475.

4. Al-Maslamani Y, Muhsin ASA, Ali OIM, et al: Potential brain death organ donors - challenges and prospects: a single center retrospective review. Saudi J Kidney Dis Transpl 2014;25(3): 589596.

5. Morgan M, Kenten C, Deedat S, et al: Increasing the acceptability and rates of organ donation among minority ethnic groups: a program of observational and evaluative research on donation, transplantation and ethnicity (DonaTE) [Internet]. South hempton UK: NIHR Journal library 2016 Mar.Programme Grants Appl Res No: 4.4. Available from: https://www.ncbi.nlm.nih.gov/books/NBK355264. DOI: 10.3310/pgfar04040.

6. Ralph AF, Alyami A, Allen RDM, Howard K, et al: Attitudes and beliefs about deceased organ donation in the Arabic-speaking community in Australia: a focus group study. BMJ Open [Internet]. Available form: http://bmjopen.bmj.com/content/6/1/e010138. DOI: 10.1136/bmjopen2015-010138.

7. El-Shoubaki H, Bener A, Al-Mosalamani Y: Factors influencing organ donation and transplantation in State of Qatar. Transplantationsmedizin 2006;18: 97-103.

8. Al Jazeera Media Network: Finding organ donors among Qatar's Muslim community. 2015 [Internet] . Available from: http://www.aljazeera.com/news/2015/07/finding-organ-donors-qatarmuslim-community-150713090541298.html (accessed October 26, 2016).

9. Bener A, Zirie M, Janahi IM, et al: Prevalence of diagnosed and undiagnosed diabetes mellitus and its risk factors in a population based study of Qatar. Diabetes Res Clin Pract 2009;84: 99-106.

10. Ajzen I: The theory of planned behavior. Journal of Organizational Behavior and Human Decision Process 1991;50: 179-211.

11. Lester D: Organ donation and the fear of death. Psychol Rep DOI: 10.2466/pr0.96.3.769-770.

12. Strenge H: Fear of death and willingness to consider organ donation among medical students. Psychother Psychosom Med Psychol 1999;49(1): 23-28.

13. Irving MJ, Tong A, Jan S, et al: Factors that influence the decision to be an organ donor: a systematic review of the qualitative literature. Nephrol Dial Transplant 2012;27: 25-33.

14. The Statesman's Yearbook 2017: The Politics, Cultures and Economies of the World. United Kingdom, Palgrave Macmillan, 2017. Pg 985. ISBN 10: 1137440082 / ISBN 13: 9781137440082 , 2016. Ajzen I: The theory of planned behavior; in Lange PAM, Kruglanski W, Higgins ET (eds): Handbook of Theories of Social Psychology. London, Sage, 2012, vol 1, pp 438-459.

15. Ajzen I: The theory of planned behavior;in Lange PAM, Kruglanski W, Higgins ET(eds): Handbook of Theories of Social Psychology. London, Sage, 2012, Vol.1, pp 438-459.

16. NHSBT Organ Donation: Understanding current attitudes and behaviors towards organ donation within England. 2013 August. Available from: http://www.nhsbt.nhs.uk/to2020/thestrategy/supporting-documents/nhsbt-organ-donor-report-140813.pdf (accessed October 28, 2016).

17. Hamad Medical Corporation: Law No. (15) of 2015 on regulating the human organs transfer and transplantation. 2015. Cited December 28, 2016. Available from: https://www.hamad.qa/EN/your\%20health/Organ-Donation/Documents/Law\%2015\%20\%20English.pdf

18. Bresnahan M, Lee SY, Smith SW, Shearman S, et al: A theory of planned behavior study of college students' intention to register as organ donors in Japan, Korea, and the United States. Health Commun. 2007;21(3): 201-211.

19. Kopfman JE, Smith SW: Understanding the audiences of a health communication campaign: a discriminant analysis of potential organ donors based on intent to donate. Journal of Applied Communication Research 1996;24: 33-49. 
20. Middle East Health Magazine: Brain death legislation and organ transplantation in the Islamic world by Khan A. 2009 [Internet]. http://www.middleeasthealthmag.com/cgi-

bin/index.cgi?http://www.middleeasthealthmag.com/sep2009/feature4.htm (accessed October 25, 2016).

21. Connaughton DM, Harmon G, Cooney A, et al: The Irish living kidney donor program- why potential donors do not proceed to living kidney donation? Clin Transplant 2016;30: 17-25.

22. Trevitt R, Whittaker C, Ball EA, et al: Drop-out rate during living donor selection. EDTNA/ERCA Journal 2012;27:88-91. DOI:10.1111/j.17556686.2001.tb00148. 